\title{
Design of Digital Testbeds for Undergraduate Microelectronics Teaching
}

\author{
Matthew F. Brejza, Jeffrey Hooker, Jonathan Sowman, David Oakley, Robert G. Maunder \\ Department of Electronics and Computer Science, University of Southampton, SO17 1BJ, UK \\ Email: $\{\mathrm{mfb} 2 \mathrm{~g} 09$, jh1, do, rm $\} @$ ecs.soton.ac.uk, j.sowman@ @oton.ac.uk
}

\begin{abstract}
In this paper we detail the series of digital microelectronics testbed boards which have been developed to meet the requirements of the microelectronics undergraduate programmes at the University of Southampton. We discuss how our boards solve many of the issues with the digital testbeds used previously for the microelectronics programmes and those which are available on the market. The digital testbed solution described in this paper is modular, and comprises multiple boards which each perform a specific function. This modular design is compact and easily expandable, while being cost effective such that they can be given to students to use at home. The boards are well suited for use with modern digital components, and fit in well with teaching of microcontrollers, programmable logic, and discrete logic.
\end{abstract}

\section{INTRODUCTION}

Digital microelectronics features heavily in modern microelectronics teaching, which requires appropriate equipment to provide inputs to circuits and monitor outputs. Typically, this takes the form of a 'digital testbed,' which will typically comprise digital inputs driven by switches, Light Emitting Diodes (LEDs) to show the status of outputs, and a square wave generator for use as a clock. For the microelectronics programmes at Southampton [1], the existing aging digital testbeds were in need of being replaced. Although replacements are available commercially and aimed at the microelectronics teaching market, they were deemed unsuitable for the programme at the University of Southampton, and so a custom solution was developed, which is also available for others to use [2].

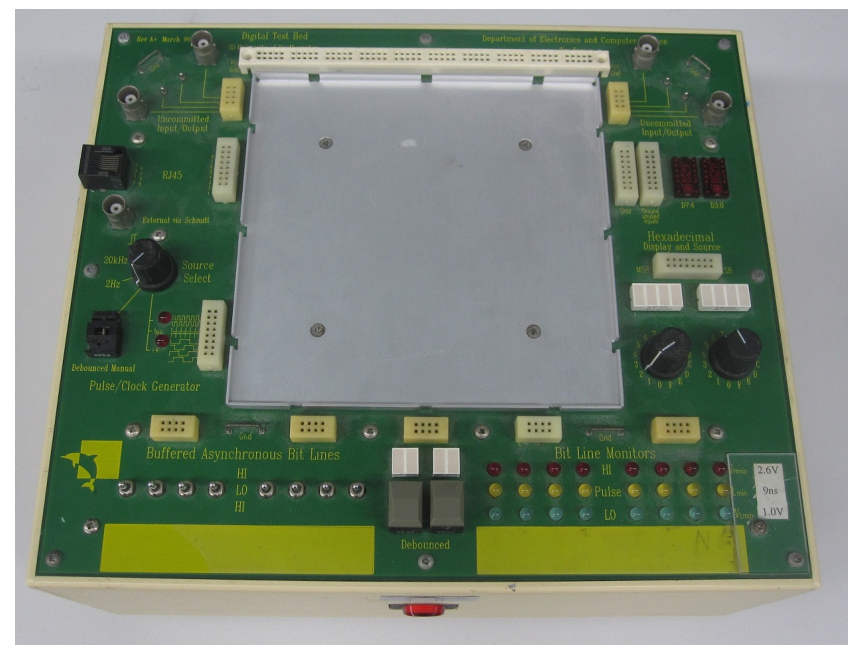

Fig. 1: The digital testbed previously used for the microelectronics programmes at the University of Southampton, which is also typical of testbeds used at other universities.
Figure 1 shows the digital testbed which has been used for the microelectronics programme since 1996. This custom solution, as well as commercial solutions, suffer from a series of issues which were addressed by the boards described in this paper. Firstly, the existing testbeds have failed to keep pace with the microelectronics industry. More specifically, the majority of new digital parts operate with a supply voltage of $3.3 \mathrm{~V}$, while most digital testbeds operate at $5 \mathrm{~V}$, as used by older digital systems. Secondly, the existing testbeds are not modular. This means the number of inputs or outputs of the digital testbed impose limits on what the students build. If more inputs or outputs are required then another testbed unit is required. Since many testbed units are bulky this may not be convenient. Furthermore, should a testbed fail, then they are typically difficult to fix, and the entire unit may need replacing rather than the specific part which has broken. Finally, by developing a testbed solution specific to the programme content, it can better serve the requirements for that programme. We were also able to create low cost options, which can be given away to the students to use at home [3]. This enables the students to pursue microelectronics outside of their studies, allowing them to develop a passion for microelectronics that can be reinvested into their studies [4].

This paper starts in Section II by detailing the requirements which the developed boards had to meet, followed by a brief discussion of each board in Section III. Section IV describes how they are used in the lab environment, and we conclude in Section V.

\section{REQUIREMENTS OF THE BOARDS}

In this section we detail the requirements of the boards which address the issues discussed in Section I, and also address the specific requirements of the programme at Southampton. To address many of the issues, the requirements dictated a solution that was fundamentally different to existing ones. Instead of one large unit, the testbed was designed to comprise multiple, modular units. More specifically, separate boards were designed for each of the different parts of the testbed, where each board fulfills only one function. This addresses many of the issues with existing units. Firstly, it provides flexibility since more boards can be added to increase the size of the testbed as required to provide more inputs or outputs. This would be impracticable with conventional testbeds due to the size and cost of the units. Furthermore, if one board is damaged, which is to be expected from an undergraduate teaching environment, it can be quickly switched out and changed without having to replace or repair a larger unit. Additionally, the modularity means the testbeds are less bulky, and can be arranged on the desk in a convenient manner. 


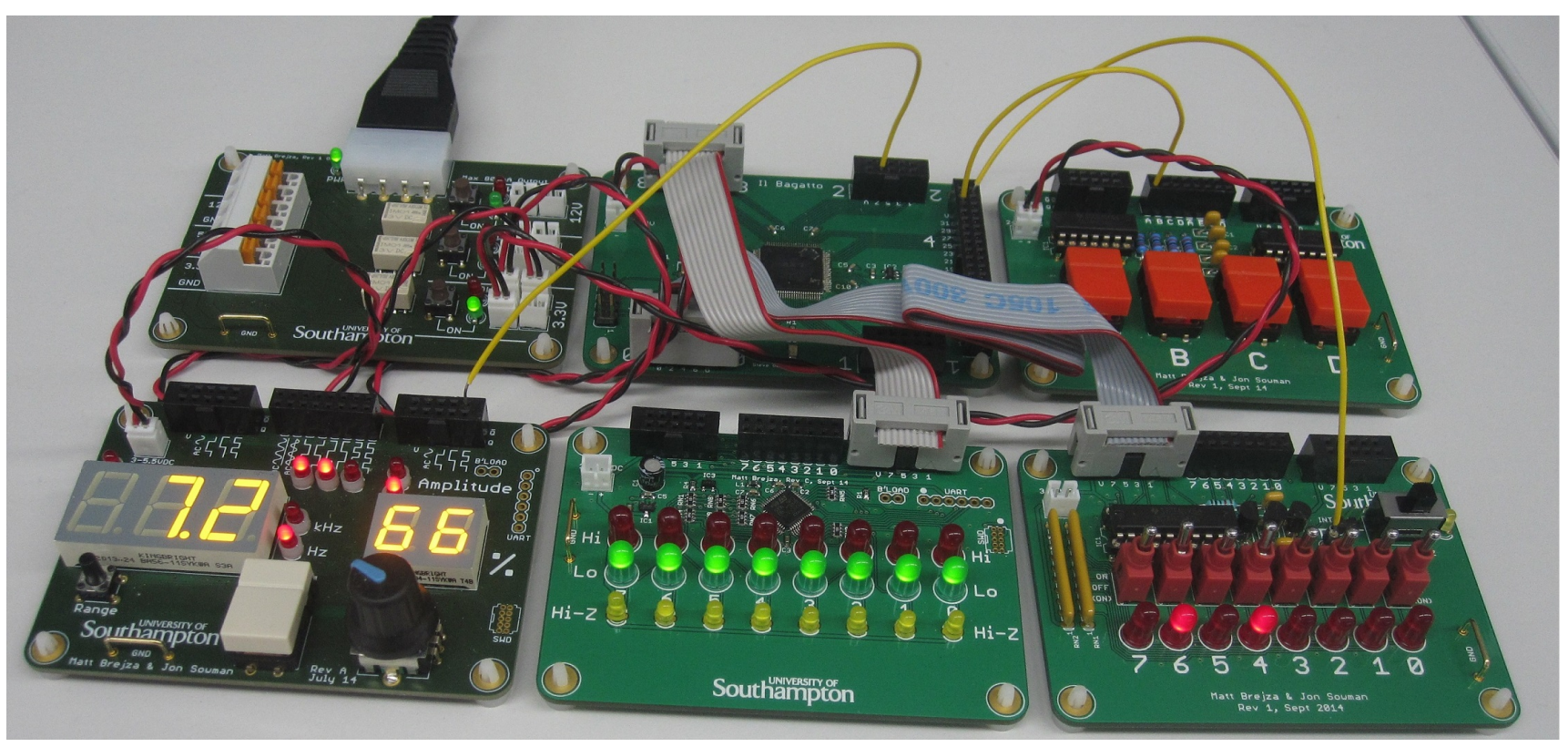

Fig. 2: The boards connected together with the CPLD board for an exercise. The boards are (clockwise from the top left) the power board, the CPLD board, the debounced button board, the slide switch board, the LED board and the clock generator board. The boards are connected together by a mix of ribbon cables and individual wires. Power is provided from the power board to the rest of the boards.

The testbed boards were also designed to work with existing boards used in the lab. More specifically, an AVR microcontroller board and an Altera Complex Programmable Logic Device (CPLD) [1] board are used in laboratory teaching and these boards share similar connectors for power and data. The digital testbed boards therefore share these similar connectors, allowing connections between boards to be made quickly, and to give the students familiarity between different boards. The CPLD board is shown in Figure 2 alongside the digital testbed boards. The digital testbed boards were required to be producible at low cost and in-house, so that they can be distributed as kits to students alongside the existing microcontroller and CPLD boards.

\section{BOARD FEATURES}

In this section we describe the features shared by all of our digital testbed boards, followed by a description of the features of each specific boards, namely the LED board, the debounced button board, the slide switch board, the clock generator board and the power board.

\section{A. General Features}

Figure 2 shows each of the different digital testbed boards, which are being used to undertake a CPLD exercise. The digital testbed boards are all the same size, which match other boards used in the lab to provide a constant look and feel between the boards. The connectors are also consistent between all the boards used in the lab. More specifically, for digital signals, each board features keyed 10-pin connectors which provide an 8-bit bus, as well as power and ground. Figure 2 shows that these connectors allow different boards to be very quickly connected together using an appropriate ribbon cable. This figure also shows how switches can provide eight inputs to one connector on the CPLD board, while LEDs can monitor another eight outputs of the CPLD board. Wires can also be inserted into the 10 pin connectors to allow connection to a breadboard or other boards. For example, in Figure 2, wires are used to provide the CPLD with a clock signal from the clock board, and a reset signal from the debounced button board. For convenience, two 10pin connectors are provided on the digital testbed boards which allow daisy-chaining, while the eight connections are also duplicated on a central 8-way connector.

To match the power requirements of typical modern digital ICs, each board can operate throughout the range of at least $3.3 \mathrm{~V}-5 \mathrm{~V}$. This is in contrast to existing systems which operate at $5 \mathrm{~V}$ only. Power can be supplied to each board by a 'JST PH' connector, or power can be shared through the 10-pin connectors.

\section{B. LED Board}

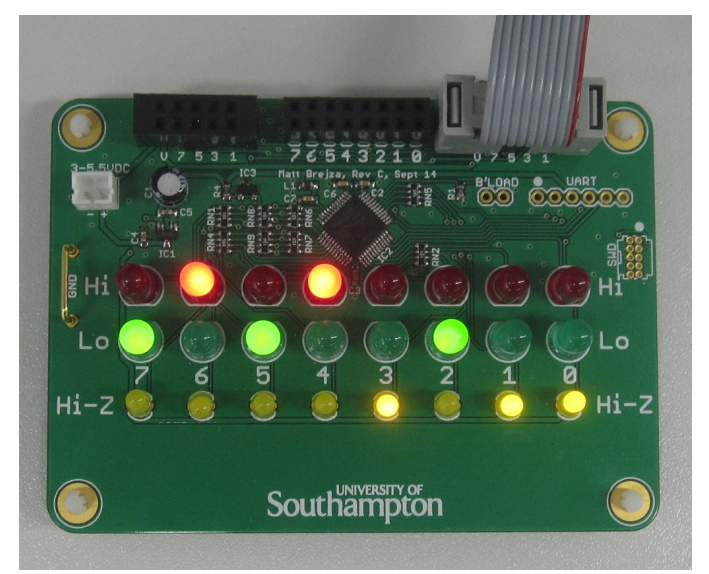

Fig. 3: The LED board

The LED board shown in Figure 3 displays the state of the eight inputs, and indicates whether each input is high, low, or high-impedance (Hi-Z). It is powered by a single 
ARM Cortex-M0 microcontroller, which rapidly samples each input and controls each LED. The addition of a Hi-Z LED, which is not found on many other solutions, removes the ambiguity between a signal which is low and a signal which is Hi-Z or unconnected. This gives better indication to students on the state of their system, which is particularly valuable in scenarios which include tri-state buses.

\section{Debounced Buttons}

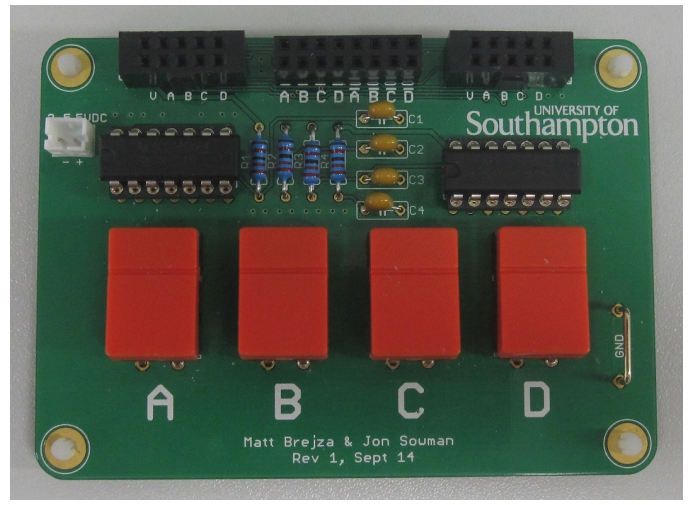

Fig. 4: The debounced switches board

Figure 4 shows the debounced switch board which comprises four debounced push buttons, that provide four normally-low outputs and four complementary normallyhigh outputs. This board is useful for providing reset signals or other momentary signals. This board uses through-hole components only, so it can be easily assembled as a kit by students.

\section{Slide Switches}

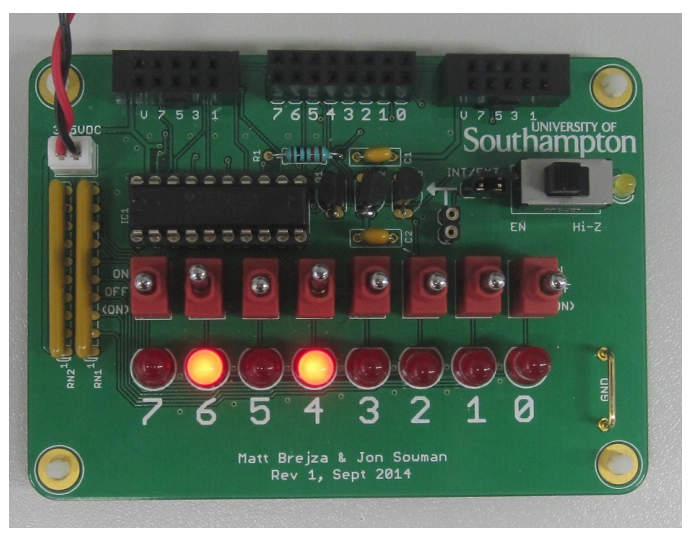

Fig. 5: The slide switches board

This board has eight slide switches which drive outputs of the board, as shown in Figure 5. This board can also operate on tri-state buses since there is also a Hi-Z switch to disable the outputs. This Hi-Z signal can be daisy chained to other boards, which also allows the Hi-Z operation to be controlled from another boards, such as another slide switch board or from the circuit the student has constructed. Figure 2 shows this in operation, where the Hi-Z operation is controlled from the CPLD board. Each switch has an LED to show when that output is high, and an LED to show when the outputs are high impedance. This board also uses through-hole components so can easily be assembled as a kit by students.

\section{E. Clock Generator}

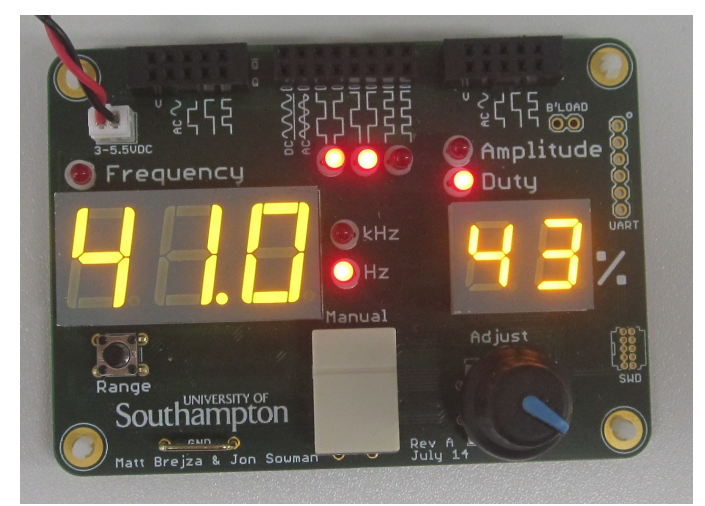

Fig. 6: The clock generator board

This board, shown in Figure 6, can provide a programmable clock signal from $0.1 \mathrm{~Hz}$ to $1 \mathrm{MHz}$. The frequency and duty cycle can be changed using the rotary encoder. The board provides the standard output as well as quadrature outputs, and their complements. The board can switch between running at the selected frequency, or manual clocking when the user presses the button at the bottom. This allows flexibility when testing circuits. The board also has a sine wave generator operating at the same frequency as the main outputs, and with variable amplitude. This analogue output is generated by the same ARM CortexM0 microcontroller which operates the rest of the board. While the lab contains dedicated function generators, this analogue output is aimed at encouraging home use rather than during university labs.

\section{F. Power Board}

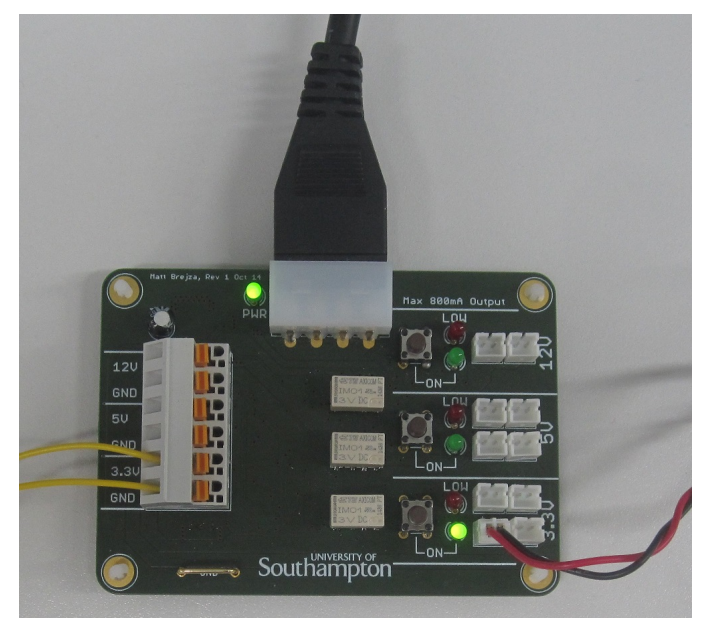

Fig. 7: The power board

Figure 7 shows the power board, which may be used to distribute power to the rest of the boards. It provides three output voltages $(12 \mathrm{~V}, 5 \mathrm{~V}$ and $3.3 \mathrm{~V})$, which are available by inserting wire into spring contacts, as shown in Figure 7, or by using the JST connectors which all the boards have in common, as shown in Figure 2. The power board can turn on or off each voltage rail, and it also monitors each voltage rail, indicating fault conditions such as short circuits. The power board itself is powered by a standard mains power 
supply which has a common 4-pin molex connector, and is protected from overcurrent by resettable fuses.

\section{LABORATORY USE}

The boards have gradually been introduced to existing first- and second-year undergraduate labs over the last year. They have been adopted in labs for discrete logic design, Programmable Logic Device (PLD) and CPLD exercises, as well as AVR programming exercises.

A notable lab in the first year is the the CPLD design exercise. This tasks the students to develop and extend a simple microprocessor and run it on a CPLD. They are given a design which can perform a few instructions, but are tasked with extending it to perform more advanced operations. The microprocessor is written in Verilog, and they are first required to test it in Modelsim, before synthesizing it for a CPLD. During the testing of the design, the clock board may be used to generate a variable frequency clock, where the manual clocking mode is useful for stepping through clock cycle by clock cycle to compare the value of the main bus with that shown in simulation. The LED board is useful for viewing the state of the tri-state bus due to the Hi-Z LEDs. The slide switches were used to provide external inputs to the microprocessor, either by being connected to separate pins on the CPLD board, or by direct connection to the tri-state bus. Students are then able to control the tri-state operation of the slide switches from an additional output signal from the CPLD. The students were free to extend the microprocessor as they wished, and made use of additional boards as they required. Some students used an additional slide switch board to provide a 16-bit input to their microprocessor, while some used an additional LED board to provide a memory-mapped output as well as monitoring the status of the tri-state bus.

\section{CONCLUSIONS}

The digital testbed boards have succeeded in replacing the existing aging units, and filled the requirements for a modular system that is well suited for modern digital microelectronics. They have been well received by students and staff in Southampton, and at the University of Southampton Malaysia Campus (USMC).

\section{REFERENCES}

[1] R. G. Maunder, "Innovation in the Undergraduate Microelectronics Programmes of the University of Southampton," in European Workshop on Microelectronics Education, Southampton, 2016 (Submitted).

[2] M. F. Brejza, J. Sowman, J. Hooker, and D. Oakley, "Digital Testbeds for Undergraduate Microelectronics Teaching - Design Files," 2016. [Online]. Available: http://eprints.soton.ac.uk/387272/

[3] J. Long, J. Florance, and M. Joordens, "The use of home experimentation kits for distance students in first-year undergraduate electronics," Proceedings of the 2004 American Society for Engineering Education Annual Conference \& Exposition, 2004. [Online]. Available: http://dro.deakin.edu.au/view/DU:30005458

[4] P. van Kollenburg and D. van Schenk Brill, "Motivating Students in Engineering \& ICT Education," in SEFI Annual Conference, Rotterdam, 2009. 